\title{
High resolution SPECT imaging for visualization of intratumoral heterogeneity using a SPECT/CT scanner dedicated for small animal imaging
}

\author{
Izumi O. Umeda $\cdot$ Kotaro Tani $\cdot$ Keisuke Tsuda $\cdot$ Masamitsu Kobayashi $\cdot$ \\ Mayumi Ogata $\cdot$ Sadaaki Kimura $\cdot$ Mitsuyoshi Yoshimoto $\cdot$ Shuji Kojima \\ Kunikazu Moribe $\cdot$ Keiji Yamamoto $\cdot$ Noriyuki Moriyama $\cdot$ Hirofumi Fujii
}

Received: 4 September 2011/Accepted: 21 September 2011/Published online: 12 October 2011

(C) The Japanese Society of Nuclear Medicine 2011

\begin{abstract}
Objectives Tumor interiors are never homogeneous and in vivo visualization of intratumoral heterogeneity would be an innovation that contributes to improved cancer therapy. But, conventional nuclear medicine tests have failed to visualize heterogeneity in vivo because of limited spatial resolution. Recently developed single photon emission computed tomographic (SPECT) scanners dedicated for small animal imaging are of interest due to their excellent spatial resolution of $<1 \mathrm{~mm}$, but few studies have focused on the evaluation of intratumoral heterogeneity. We investigated the optimal conditions related to high resolution imaging of heterogeneous tumor interiors using a small animal SPECT scanner.
\end{abstract}

I. O. Umeda $(\bowtie) \cdot$ K. Tani $\cdot$ K. Tsuda $\cdot$ M. Kobayashi

M. Ogata $\cdot$ S. Kimura $\cdot$ H. Fujii

Functional Imaging Division, Research Center for Innovative

Oncology, National Cancer Center Hospital East,

6-5-1 Kashiwanoha, Kashiwa, Chiba 277-8577, Japan

e-mail: ioumeda@east.ncc.go.jp

M. Kobayashi · S. Kojima

Faculty of Pharmaceutical Sciences,

Tokyo University of Science,

2641 Yamazaki, Noda, Chiba 278-8510, Japan

M. Ogata $\cdot$ K. Moribe $\cdot$ K. Yamamoto

Graduate School of Pharmaceutical Sciences, Chiba University,

1-8-1 Inohana, Chuo-ku, Chiba 260-8675, Japan

M. Yoshimoto

Division of Cancer Development System, National Cancer

Center Research Center, 5-1-1 Tsukiji, Chuo-ku,

Tokyo 104-0045, Japan

\section{N. Moriyama}

Research Center for Cancer Prevention and Screening,

National Cancer Center, 1-1 Tsukiji, Chuo-ku,

Tokyo 104-0045, Japan
Methods The conditions related to SPECT/CT visualization of heterogeneous tumor interiors were investigated using phantoms with ${ }^{111}$ In and simulations of actual small animal imaging. The optimal conditions obtained were validated by in vivo imaging of sarcoma 180-bearing mice. Results Larger number of counts must be obtained within limited acquisition time to visualize tumor heterogeneity in vivo in animal imaging, compared to cases that simply detect tumors. At an acquisition time of $30 \mathrm{~min}$, better image quality was obtained with pinhole apertures diameter of $1.4 \mathrm{~mm}$ than of $1.0 \mathrm{~mm}$. The obtained best spatial resolution was $1.3 \mathrm{~mm}$, it was acceptable for our purpose, though a little worse than the best possible performance of the scanner $(1.0 \mathrm{~mm})$. Additionally, the reconstruction parameters, such as noise suppression, voxel size, and iteration/subset number, needed to be optimized under the limited conditions and were different from those found under the ideal condition. The minimal radioactivity concentration for visualization of heterogeneous tumor interiors was estimated to be as high as $0.2-0.5 \mathrm{MBq} / \mathrm{mL}$. Liposomes containing ${ }^{111}$ In met this requirement and were administered to tumor-bearing mice. SPECT imaging successfully showed heterogeneous ${ }^{111}$ In distribution within the tumors in vivo with good spatial resolution. A threshold of $0.2 \mathrm{MBq} / \mathrm{g}$ for clear visualization of tumor heterogeneity was validated. Autoradiograms obtained ex vivo of excised tumors confirmed that the in vivo SPECT images accurately depicted the heterogeneous intratumoral accumulation of liposomes.

Conclusion Intratumoral heterogeneity was successfully visualized under the optimized conditions using a SPECT/ CT scanner.

Keywords Small animal - SPECT · Tumor heterogeneity $\cdot{ }^{111} \mathrm{In} \cdot$ Liposome 


\section{Introduction}

Radionuclide imaging such as positron emission tomography (PET) and single-photon emission computed tomography (SPECT) can visualize in vivo physiological function even in deep sites of the body with high sensitivity and excellent quantitative capability and are powerful tools for cancer imaging [1]. These techniques can detect tumor masses in vivo, but they cannot visualize interiors of tumor masses because the spatial resolution of conventional radionuclide imaging devices is quite poor ( $>5 \mathrm{~mm}$ for PET and $>8 \mathrm{~mm}$ for SPECT). However, tumors are never homogeneous. Therefore, a technique with the ability to clearly visualize intratumoral heterogeneity in vivo would be an innovation that improves cancer diagnosis and therapy.

Recently, SPECT scanners dedicated for small animal imaging have been developed and have shown excellent spatial resolution of $<1 \mathrm{~mm}$ [2-5]. They appear to hold potential for visualization of intratumoral heterogeneity in vivo. However, most studies on high spatial resolution using these scanners have been conducted with phantoms under ideal conditions [6-8], and little work has been done with actual small animal imaging. A few studies have reported in vivo SPECT images of small animals with excellent resolution [2, 9]; however, the administered radioactivity in these studies was approximately $200-900 \mathrm{MBq}$ per head using ${ }^{99 \mathrm{~m}} \mathrm{Tc}$ - or ${ }^{123} \mathrm{I}$-labeled probes. This dosage is very high for actual small animal imaging, and hence, is likely to induce very high radiation in the animals [10]. On the other hand, very recently, increasing numbers of studies have reported the use of these scanners for tumor imaging of small animals [4, 11-14]. The administered radioactivity used in these studies seems to be reasonable for small animals, but instead, they visualized just tumor masses, not tumor interior, because of insufficient spatial resolution. Although a few studies have reported good resolution [11, 12], they were not necessarily optimized for in vivo high resolution tumor imaging. In this study, we investigated the acceptable and optimal conditions related to high resolution in vivo SPECT imaging for actual small animals, such as acquisition equipment and time, reconstruction parameters, and detection and dosage thresholds for visualization of intratumoral heterogeneity in vivo. We also used this technique to visualize intratumoral heterogeneity in tumor-bearing mice with fine spatial resolution using a small animal SPECT scanner.

\section{Materials and methods}

\section{SPECT/CT imaging}

A SPECT/CT combined scanner with four detectors dedicated for small animal imaging (NanoSPECT/CT; Bioscan
Inc., Washington, DC, USA) was used to acquire sectional images. Each detector was equipped with tungsten-based multiplexing nine-pinhole collimators with diameters of 1.0 or $1.4 \mathrm{~mm}$ and a cylindrical field of view (FOV) of $30 \times 16 \mathrm{~mm}$ (diameter $\times$ length). The axial FOV was extended using a step-and-shoot helical SPECT with a user-definable range up to $230 \mathrm{~mm}$, depending on the imaging region. The energy peaks for the camera were set to 171 and $245 \mathrm{keV}$, and the energy window was set to the peak energy $\pm 10 \%$. CT scans were performed with the following conditions: tube voltage, $45 \mathrm{kV}$; tube current, $177 \mu \mathrm{A}$; and tube current-time product (the product of the tube current and scan time), $32 \mathrm{mAs}$. CT and SPECT reconstruction was performed using InVivoScope software (Bioscan). CT images were reconstructed with the standard resolution, and SPECT image data were reconstructed by an ordered subset expectation maximization iterative algorithm dedicated for multiplexing multi-pinhole reconstruction. Three operator-dependent parameters were used during the reconstruction of the SPECT image data. They were noise suppression (low, middle, and high), voxel size (fast $0.4 \mathrm{~mm}$; standard $0.3 \mathrm{~mm}$; and fine $0.2 \mathrm{~mm}$ ), and a reconstruction setting for the iteration/subset number (fast, standard, and fine). The following two sets of reconstruction parameters (conditions A and B) were used in this study. For noise suppression, voxel size, and reconstruction setting, the values were low ( $25 \%$ of voxel size), fine, and fine for condition A, respectively, and middle (35\%), standard, and standard for condition B, respectively. The SPECT/CT fusion images were obtained using the automatic fusion software installed on the scanner.

\section{Phantom studies}

System performance evaluation by measurement of the spatial resolution and sensitivity of the SPECT scanner

A Jaszczak phantom with hot rods ranging in diameter from 1.0 to $1.5 \mathrm{~mm}$ (Bioscan), which was filled with $100 \mathrm{MBq}$ $\left(50 \mathrm{MBq} / \mathrm{mL}\right.$ ) of ${ }^{111} \mathrm{InCl}_{3}$ solution (kind gift by Nihon MediPhysics, Tokyo, Japan), was imaged according to the procedure described above, using the SPECT scanner equipped with 1.0- and 1.4-mm diameter pinhole apertures. Projection image data were acquired in the step-and-shoot mode from 128 directions over $360^{\circ}$ covered by four detectors (32 directions $\times 4$ detectors). The total acquisition count was set at 300 Mcounts. Consequently, the total acquisition time was several hours. The acquired image data were reconstructed using conditions A and B mentioned above. The reconstructed sectional images were evaluated on the basis of count profile curve methods. When the smallest count in the intercolumn space was lower than $50 \%$ of the maximum 
count of the columns, we judged that the columns were separately imaged. Additionally, the smallest diameter at which each column was separately imaged was defined as the spatial resolution. The sensitivity of each aperture for ${ }^{111} \mathrm{In}$ was measured using an ${ }^{111}$ In point source. Three MBq of

${ }^{111}$ In was absorbed into a 0.15 - to $0.30-\mathrm{mm}$ resin bead, which was placed at the center of the FOV and imaged from 128 $(32 \times 4)$ projections with an acquisition time of $30 \mathrm{~s}$ per projection. Sensitivity was calculated by dividing the acquired counts per second (cps) by the radioactivity of the point source $(\mathrm{Bq})$. The acquisition was repeated three times.

\section{Measurement of the SPECT image spatial resolution} under the conditions simulated conditions of small animal imaging

For simulation of actual in vivo animal imaging, a Jaszczak phantom with rods of 1.0 - to $1.5-\mathrm{mm}$ diameter was filled with $10 \mathrm{MBq}(5 \mathrm{MBq} / \mathrm{mL})$ of ${ }^{111} \mathrm{InCl}_{3}$ solution. SPECT image data were acquired in the similar manner to mentioned above, except projection numbers, that is, 24 projections over $360^{\circ}$ covered by four detectors $(6 \times 4)$. The total acquisition time was set as $5-120 \mathrm{~min}$. The obtained image data were reconstructed in the same manner as described above.

\section{Evaluation of the lower limit for visualization and quantitative linearity of the SPECT images}

Six 1-mL plastic syringes with an inner diameter of $4.3 \mathrm{~mm}$ were filled with ${ }^{111} \mathrm{InCl}_{3}$ solution with a concentration between 0 and $5.0 \mathrm{MBq} / \mathrm{mL}$, and the image data were acquired in the same manner as above from 24 $(6 \times 4)$ views with $300 \mathrm{~s}$ per projection, consequently a total acquisition time was $30 \mathrm{~min}$. The acquired image data were reconstructed using condition $\mathrm{B}$. Regions of interest (ROIs) of the same size were placed on each syringe based on the superimposed CT images, and the radioactivity of each ROI was measured (SPECT value).

Animal studies

\section{Chemicals}

Distearoylphosphatidylcholine (DSPC; Coatsome MC8080) and cholesterol were purchased from NOF Corporation (Tokyo, Japan). Diethylenetriaminepentaacetic acid (DTPA) and 8-hydroxyquinoline were purchased from Wako Pure Chemicals (Osaka, Japan). Isoflurane was purchased from Abbott Japan (Tokyo, Japan). All other chemicals were purchased from Wako Pure Chemicals or Sigma-Aldrich Japan (Tokyo, Japan).

\section{Animals}

Male ddY mice were obtained from Japan SLC Inc. (Hamamatsu, Japan). The animals were kept at 12/12-h dark-light cycles and were fed ad libitum. Sarcoma 180 tumor cells $\left(2 \times 10^{6}\right.$ cells/mouse $)$ were subcutaneously transplanted into the left hind leg of 6- to 7-week-old mice. When the tumor weighed between 0.3 and $1.0 \mathrm{~g}$, the mice were used for the experiments. All procedures were performed in accordance with the Guidelines of the Animal Care and Use Committee of the National Cancer Center.

Preparation of ${ }^{111}$ In-labeled liposomes at high concentration

Liposomes were composed of DSPC and cholesterol at a molar ratio of 2:1 and prepared by a lipid film hydrationextrusion method [15]. In brief, the dried films were dispersed in $10 \mathrm{mM}$ DTPA in $50 \mathrm{mM}$ HEPES/5\% mannitol buffer, $\mathrm{pH} 7.4$, at $60^{\circ} \mathrm{C}$ and then repeatedly extruded through polycarbonate membrane filters (0.2-, $0.1-$, and $0.05-\mu \mathrm{m}$ pore sizes) to adjust their diameters to about $100 \mathrm{~nm}$. The extra chelating ligands were removed by passage through a Sephadex G-50 (GE Healthcare Japan Ltd., Tokyo, Japan) column. The phospholipid concentration was measured by phosphate assay. The liposomes were then labeled with ${ }^{111}$ In. To achieve a very high level of specific radioactivity, a remote loading method was used [16]; specifically, ${ }^{111}$ In was loaded inside the liposomes through the liposomal membranes by transchelation between the lipophilic oxine and hydrophilic DTPA ligands.

\section{In vivo SPECT/CT imaging with high spatial resolution}

The ${ }^{111}$ In-labeled liposomes (8.5-15 MBq/0.2 mL/mouse) were injected intravenously into sarcoma 180-bearing mice, and CT and SPECT images were acquired 24 or $48 \mathrm{~h}$ later. Mice were anesthetized by inhalation of $1.5 \%$ isoflurane, and their body temperatures were kept at about $37^{\circ} \mathrm{C}$ during the scans. Acquisition and reconstruction of the CT and SPECT image data were performed under the conditions optimized by the phantom studies simulating animal imaging. SPECT projection data were acquired in a step-and-shoot mode from $24(6 \times 4)$ views covering the whole body or the tumor site. The acquisition time was set to $150-300 \mathrm{~s}$ per projection, and image data were acquired from 24 projections taking a total of 1-2 h. The acquired projection data were reconstructed using InVivoScope software with condition B. 
Measurement of accumulated radioactivity and autoradiography $(A R G)$ of the excised tumors

After the in vivo imaging, the mice were killed, and the tumors were carefully excised. The excised tumors were immediately weighed, and their radioactivity was measured by means of a dose calibrator (IGC-7; Aloka Co., Ltd., Tokyo, Japan) or an Auto Well Gamma System (ARC380CL; Aloka). Then, the tumors were embedded in OCT compound (Sakura Finetek Japan, Tokyo, Japan), frozen and cut into $6-\mu \mathrm{m}$-thick sections for hematoxylin-eosin staining and $20-\mu \mathrm{m}$-thick sections for ARG using a cryomicrotome (CM 3050S; Leica Instruments, Germany). The sections were prepared in the same direction of SPECT slice. The section corresponding to the SPECT image was selected according to the slice thickness and used for ARG. For ARG, the samples were placed in contact with an imaging plate (MS2040; Fujifilm Co., Tokyo, Japan), and the exposed imaging plates were analyzed with an FLA7000 reader (Fujifilm Co.) and Multi Gauge v3.0 software (Fujifilm Co.).

\section{Results}

Initially, we evaluated the performance of the scanner for spatial resolution and sensitivity under ideal conditions. Figure 1 shows the SPECT images and their count profile curves of a Jaszczak phantom filled with ${ }^{111} \mathrm{InCl}_{3}$ solution as high as $50 \mathrm{MBq} / \mathrm{mL}$. Under the conditions of high radioactivity, long acquisition time, and reconstruction parameters for fine image quality (condition A), the resolution obtained was excellent. Hot rods as thin as $1.0 \mathrm{~mm}$ on the SPECT images were separately visualized using both 1.0- and 1.4-mm pinhole apertures (Fig. 1a, b). The resolution was slightly better using pinhole apertures of 1.0-mm diameter than of 1.4-mm diameter by count profile curve analysis, but there was little difference on visual evaluation. On the other hand, the sensitivity of the 1.4-mm pinhole apertures was $2.0 \mathrm{kcps} / \mathrm{MBq}$ for ${ }^{111} \mathrm{In}$, which was two times higher than that of the $1.0-\mathrm{mm}$ pinhole apertures (0.97 kcps/MBq).

The reconstruction parameters (noise suppression, voxel size, and iteration/subset number) had considerable influence on the images. Figure 1b, c shows images that were obtained from the same projection data but were reconstructed under different conditions. Figure $1 b$ was reconstructed under condition A and Fig. 1c under condition B. The condition A image showed higher resolution than the condition B image when sufficient counts were acquired, as in the system performance evaluation experiments. However, ideal acquisition conditions were not possible in the animal imaging experiments, because a large radioactive dose cannot be administered to small animals and the animal position cannot be kept stable for such a long time. Therefore, phantom experiments simulating in vivo small animal imaging were performed.

A Jaszczak phantom was filled with ${ }^{111} \mathrm{InCl}_{3}$ solution of $10 \mathrm{MBq}(5 \mathrm{MBq} / \mathrm{mL})$, and images were acquired for 5-120 min using 1.0- and 1.4-mm pinhole apertures. The acquired data were reconstructed with two different conditions, conditions A and B described above. Under these experimental conditions, images acquired with the 1.4-mm pinhole apertures were always better than those acquired with the $1.0-\mathrm{mm}$ apertures (data not shown). The images acquired with the 1.4-mm pinhole apertures are shown in Fig. 2. The SPECT images reconstructed with condition B (upper row in Fig. 2) always showed higher resolution than those reconstructed with condition A (lower row), unlike the images shown in Fig. 1b, c. Under condition B, the spatial resolution greatly depended on acquisition time, specifically, on total acquisition counts. An acquisition time of $30 \mathrm{~min}$ provided images with acceptable spatial resolution of 1.3-1.4 mm under the experimental conditions.

We also evaluated the lower limit of radioactivity for visualization. Syringe phantoms were filled with ${ }^{111} \mathrm{InCl}_{3}$ solution with concentrations of $0-5.0 \mathrm{MBq} / \mathrm{mL}$ for simulated animal imaging. Image data were acquired for $30 \mathrm{~min}$ and reconstructed using condition B. Figure 3 a shows axial images of the syringes filled with $0.1-5 \mathrm{MBq} / \mathrm{mL}$, and Fig. $3 \mathrm{~b}$ shows images of the syringes filled with $0-0.5 \mathrm{MBq} / \mathrm{mL}$. Under these conditions, the syringes with radioactivity concentrations lower than $0.1 \mathrm{MBq} / \mathrm{mL}$ could not be visualized on the reconstructed SPECT images irrespective of the scale range. Therefore, the threshold for visualization of ${ }^{111} \mathrm{In}$ was estimated to be $0.2-0.5 \mathrm{MBq} / \mathrm{mL}$ $(\mathrm{MBq} / \mathrm{g})$ under these test conditions.

The quantitative linearity of the SPECT images was also examined. ROIs of the same size were placed on each syringe based on the superimposed CT images, and their radioactivity was measured (SPECT value). Figure 3c, d shows the correlation between the radioactivity concentration of ${ }^{111} \mathrm{InCl}_{3}$ and the SPECT value. For both concentration ranges described above, including the points under $0.1 \mathrm{MBq} / \mathrm{mL}$, excellent linearity was shown $\left(R^{2}>0.99\right)$.

After optimization of the conditions needed to obtain high resolution SPECT images in vivo, we then attempted to visualize intratumoral heterogeneity. The ${ }^{111}$ In-labeled liposomes were intravenously injected into sarcoma 180 tumor-bearing mice (8-15 MBq/mouse), and SPECT/CT fusion images were obtained at $24 \mathrm{~h}$ after injection (Fig. 4). The images of case \#1 are a whole body maximum intensity projection (MIP) image and representative slice images containing the tumor. Images of case \#2 are sectional images of the tumor site of another mouse. In both cases, the ${ }^{111}$ In-labeled liposomes were strongly 
Fig. 1 SPECT images of an

${ }^{111}$ In Jaszczak phantom and their count profile curves under the best performance condition. The Jaszczak phantom was filled with ${ }^{111} \mathrm{InCl}_{3}$ of $100 \mathrm{MBq}$ $(50 \mathrm{MBq} / \mathrm{mL})$, and images were obtained on a SPECT scanner equipped with pinhole apertures with diameters of $1.0 \mathrm{~mm}$ (a) and $1.4 \mathrm{~mm}(\mathbf{b}, \mathbf{c})$.

Projection data were acquired with 128 directions over $360^{\circ}$ covered by four detectors ( 32 directions $\times 4$ detectors). Total acquisition counts were set at 300 Mcounts. The acquired projection data were reconstructed using

InVivoScope software based on ordered subset expectation maximization (OSEM) with two distinct conditions, $\mathrm{A}(\mathbf{a}, \mathbf{b})$ and B (c)

Pinhole $\boldsymbol{\varphi 1 . 0} \mathbf{~ m m}$
Condition-A
Noise suppression: Low
Voxel size: $0.2 \mathrm{~mm}$
Iteration \& subset: : Fine

\begin{tabular}{|c|c|}
\hline Pinhole $\varphi$ & $1.4 \mathrm{~mm}$ \\
\hline $\begin{array}{l}\text { Condition-A } \\
\text { Low }\end{array}$ & $\begin{array}{l}\text { Condition-B } \\
\text { Middle }\end{array}$ \\
\hline $0.2 \mathrm{~mm}$ & $0.3 \mathrm{~mm}$ \\
\hline Fine & Middle \\
\hline
\end{tabular}

Jaszczak phantom
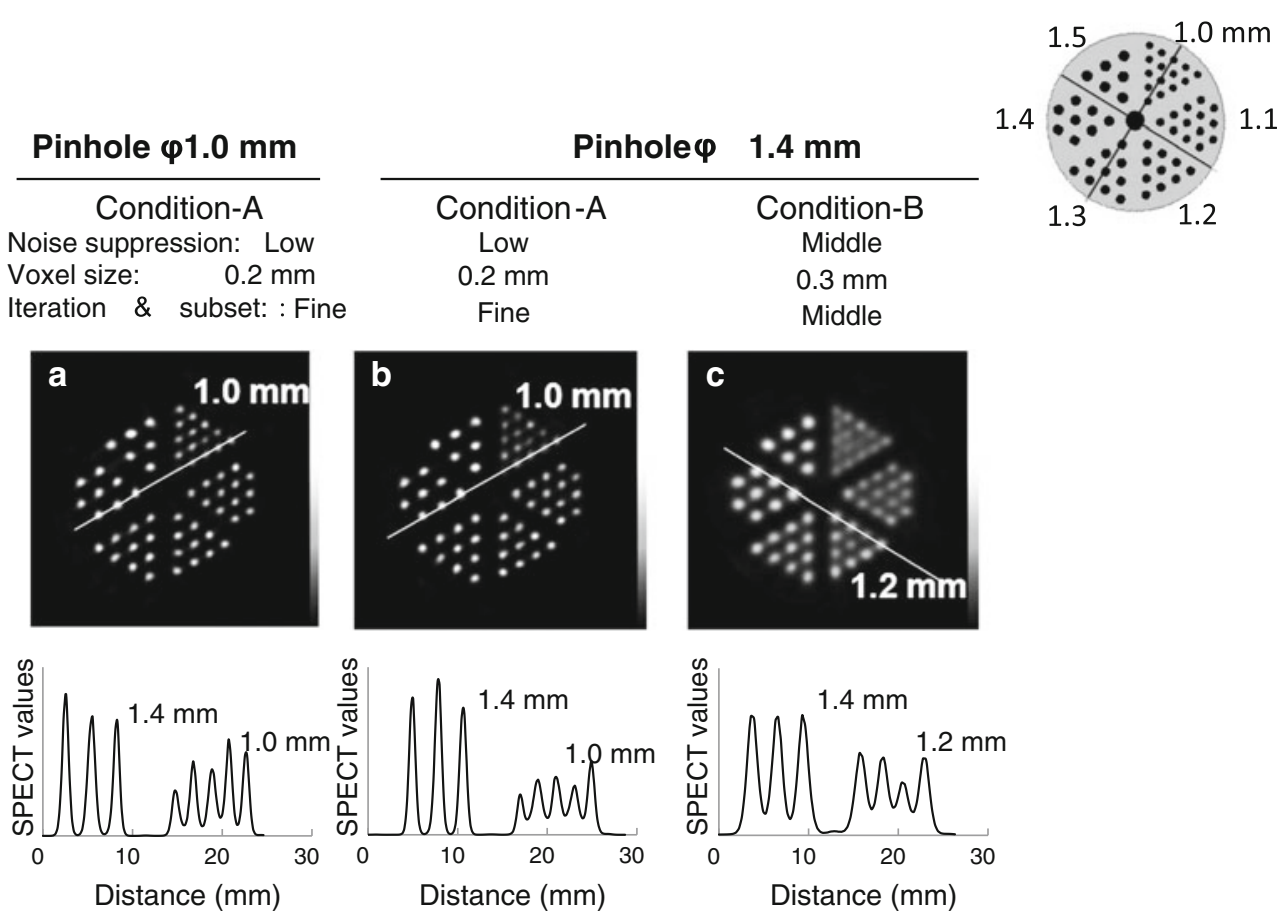
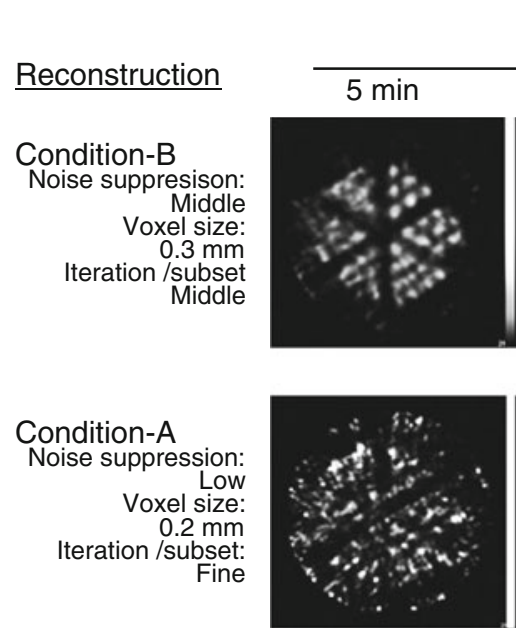
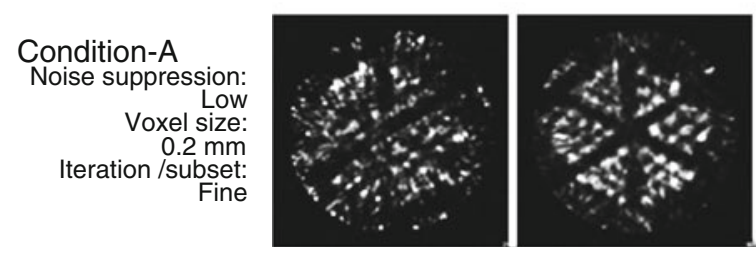

Fig. 2 SPECT images of an ${ }^{111}$ In Jaszczak phantom under conditions simulating in vivo small animal imaging. The Jaszczak phantom was filled with ${ }^{111} \mathrm{InCl}_{3}$ of $10 \mathrm{MBq}(5 \mathrm{MBq} / \mathrm{mL})$, and the images were acquired for 5-120 min with 1.4-mm pinhole apertures and 24

accumulated in the tumor, and the heterogeneous intratumoral localization in vivo was successfully visualized using SPECT with good spatial resolution. The liposomes were strongly located in the marginal regions of the tumors. We also found that the ${ }^{111}$ In-labeled liposomes were strongly accumulated in the liver and spleen as shown in the case \#1 images. These results corresponded well with our previous results for biodistribution of radioactive liposomes [15, 17-19].

Total acquisition time 
Fig. 3 The minimum radioactivity threshold for visualization and quantitative linearity of SPECT images. Six 1-mL plastic syringes were filled with ${ }^{111} \mathrm{InCl}_{3}$ of $0.1-5.0 \mathrm{MBq} / \mathrm{mL}(\mathbf{a}, \mathbf{c})$ or $0-0.5 \mathrm{MBq} / \mathrm{mL}(\mathbf{b}, \mathbf{d})$, and their SPECT images were acquired. Regions of interest of the same size were placed on each syringe based on the superimposed CT images, and the radioactivity was measured (SPECT value). a, b Axial SPECT images of the phantoms. c, d The correlation between ${ }^{111} \mathrm{InCl}_{3}$ concentration and the SPECT value
${ }^{111}$ In conc.: 0.1-5 MBq/mL
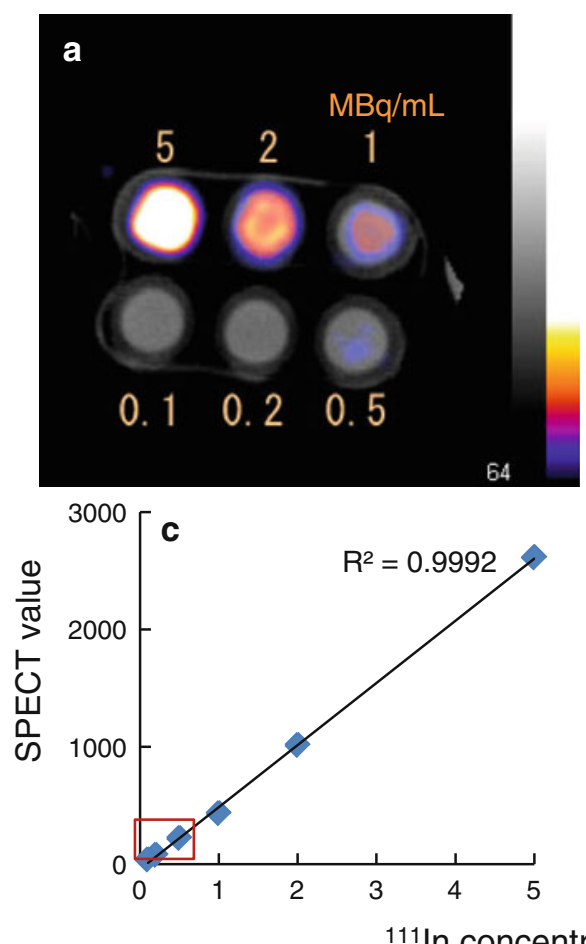

${ }^{111}$ In concentration $(\mathrm{MBq} / \mathrm{mL})$
0-0.5 MBq/mL
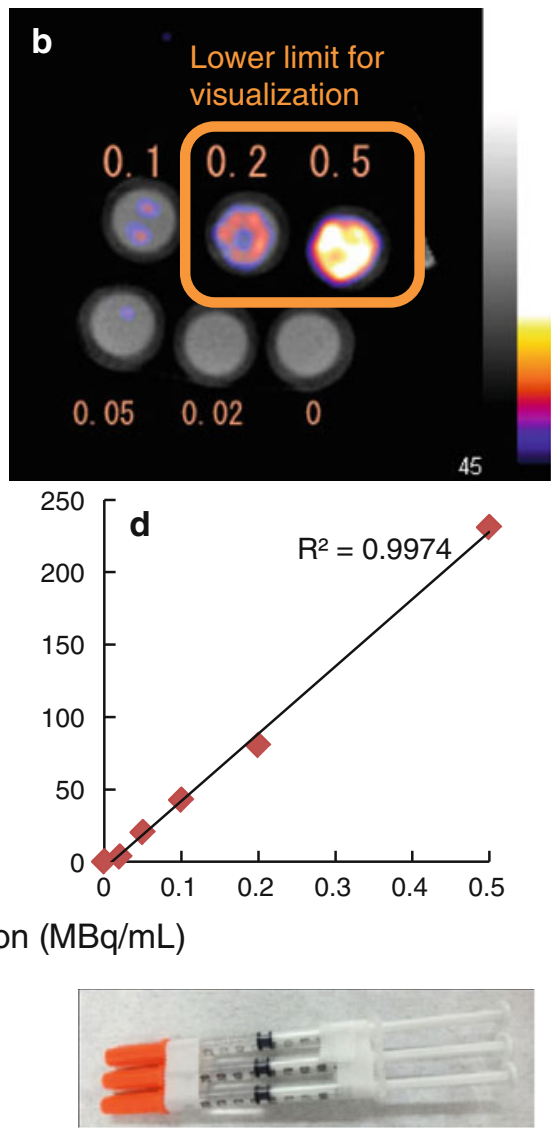

Inner diameter; $4.3 \mathrm{~mm}$
Case \#1 (Whole body Images)
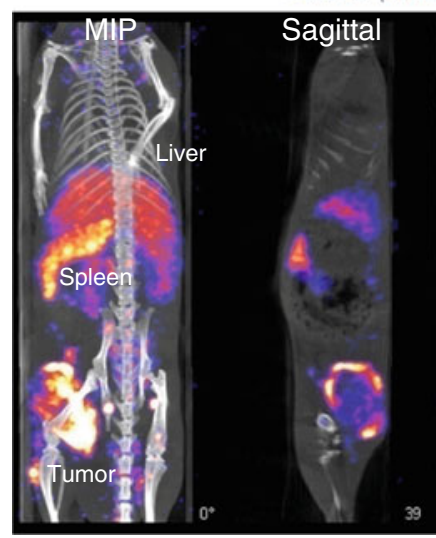

Fig. 4 In vivo SPECT/CT images of sarcoma 180-bearing mice $24 \mathrm{~h}$ after administration of ${ }^{111}$ In-labeled liposomes. Case \#1: whole body images containing maximum intensity projection (MIP) and representative sagittal, coronal, and axial slice images containing the tumor. Case \#2: representative sagittal, coronal, and axial slice

images. The ARGs showed excellent resolution and confirmed that the distribution of the ${ }^{111}$ In-labeled liposomes in the tumor was indeed heterogeneous, and in vivo SPECT images accurately reflected the heterogeneity. images of the tumor site in another mouse. The SPECT projection data were acquired under the optimized conditions for in vivo imaging. The acquisition time was $150-300$ s per projection with 24 $(6 \times 4)$ directions, total $=1-2 \mathrm{~h}$. The acquired projection data were reconstructed using InVivoScope software with condition B

Figure 6 shows in vivo axial SPECT images of varioussized tumors obtained from four individual tumor-bearing mice that were administered ${ }^{111}$ In-labeled liposomes. For every case, the intratumoral distribution could be clearly 
visualized. After the acquisition, the tumors were removed, and their weight and radioactivity were measured (Table 1). The tumor sizes were between 0.51 and $1.61 \mathrm{~g}$, and the accumulated radioactivity in the isolated tumors was between 0.26 and $1.2 \mathrm{MBq} / \mathrm{g}$ of tumor $(5-10 \%$ of the injected dose/g).

\section{Discussion}

The aim of this study was to investigate the optimal conditions required for SPECT with spatial resolution high enough to visualize intratumoral heterogeneity using in vivo small animal imaging. These approaches have not often been attempted because scanners with high spatial resolution were needed for this purpose. Conventional radionuclide imaging devices have shown quite poor spatial resolution. However, recently developed SPECT scanners dedicated for small animals excel at spatial resolution and appear to hold potential for intratumoral heterogeneity imaging applications. Our SPECT scanner provided excellent spatial resolution of $<1 \mathrm{~mm}$ in the phantom study under ideal acquisition conditions (Fig. 1a). However, it included conditions such as too high radioactivity and too long acquisition time, which are not practical in actual animal experiments. For practical purpose, most imaging studies using small animal scanners employ a more reasonable radioactive dosage and acquisition time; these conditions, however, should be insufficient for visualization of tumor heterogeneity using high resolution SPECT imaging. Therefore, in the first part of our study, we investigated the acceptable and optimal conditions required for acquisition and reconstruction of images in simulating actual animal experiments with limited radioactivity and acquisition time (Fig. 2).

As a result, we determined that adjustment of three parameters was required for optimal small animal imaging. First, we found that a pinhole aperture of $1.4 \mathrm{~mm}$ diameter was better for our purpose than an aperture of $1.0 \mathrm{~mm}$ diameter. Images from the $1.0-\mathrm{mm}$ aperture were slightly higher in resolution than those from the 1.4-mm aperture as shown in Fig. 1, but the differences were not significant by visual inspection. Evaluation of the obtained results showed that the number of acquired counts strongly affected the spatial resolution in the simulation of small animal imaging. We judged that the 1.4-mm aperture had an advantage because it gave sensitivity that was twice as high as that obtained using the $1.0-\mathrm{mm}$ aperture, as shown by the total acquired counts that were two times higher. Second, the acquisition time should be more than $30 \mathrm{~min}$ under these experimental conditions for the same reason as mentioned above (to acquire a sufficient number of counts). The time required is relatively long for in vivo animal imaging. Therefore, anesthesia and the stability of the animal position are very important. Third, the reconstruction parameters, such as noise suppression, voxel size, and iteration/subset number, should be optimized for imaging with limited acquisition counts and will be different from the parameter values required for the best system performance under ideal conditions. It is very important to choose appropriate reconstruction parameters according to the experimental objectives because the image quality varies greatly depending on the chosen values, even though the images are reconstructed from the same projection data a

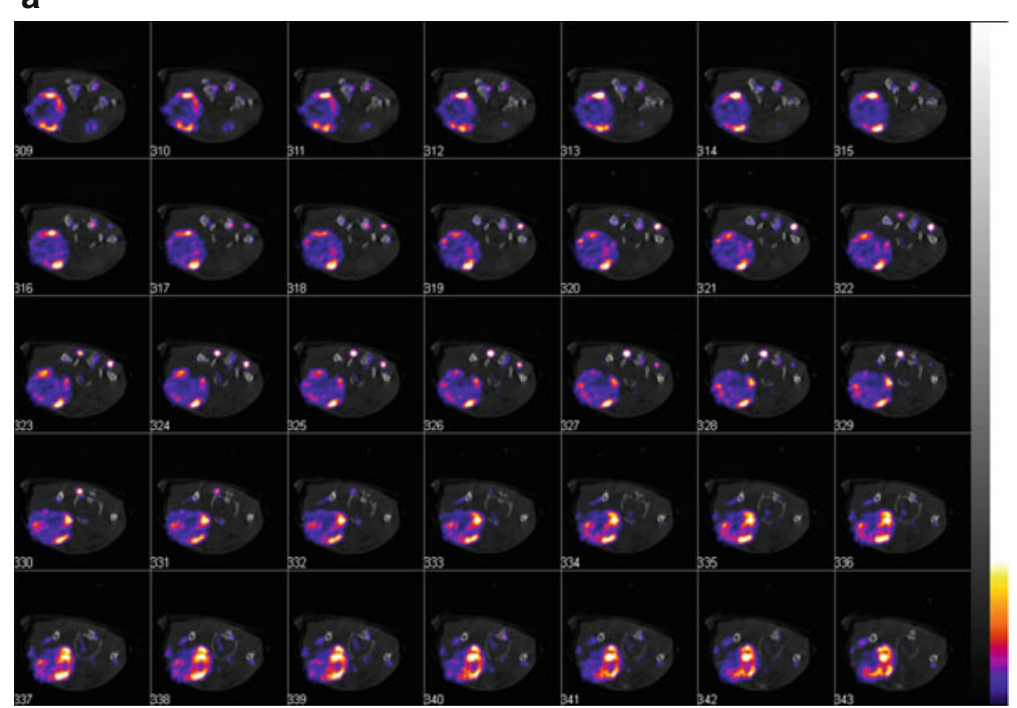

b

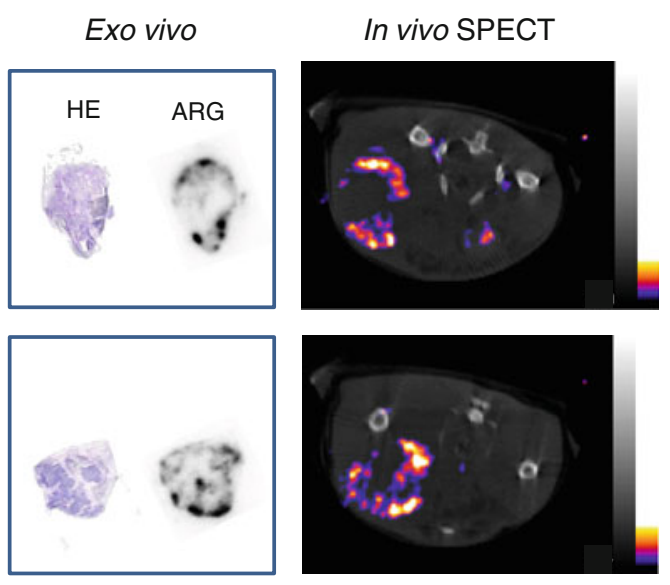

Fig. 5 Visualization of the intratumor distribution of ${ }^{111}$ In-labeled liposomes. a In vivo serial axial SPECT images of 0.2-mm thickness from the same tumor. b Ex vivo autoradiograms of excised tumor sections and the corresponding in vivo SPECT images 

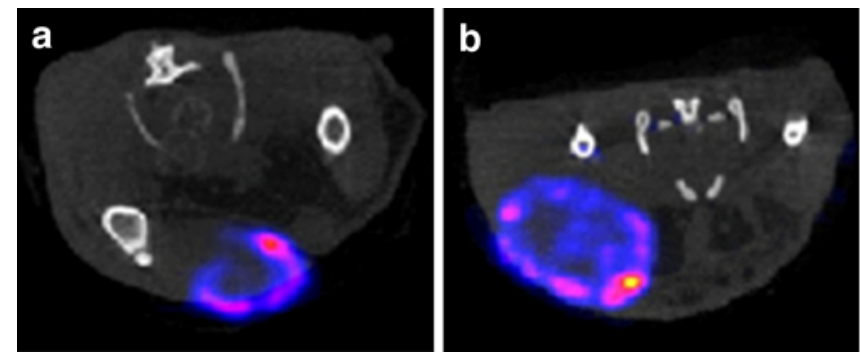

Fig. 6 Representative axial slice images of tumors obtained from four individual tumor-bearing mice administered ${ }^{111}$ In-labeled liposomes. Each image was acquired 24 or $48 \mathrm{~h}$ after administration of the ${ }^{111}$ In-labeled liposomes. The greatest dimension of the serial axial
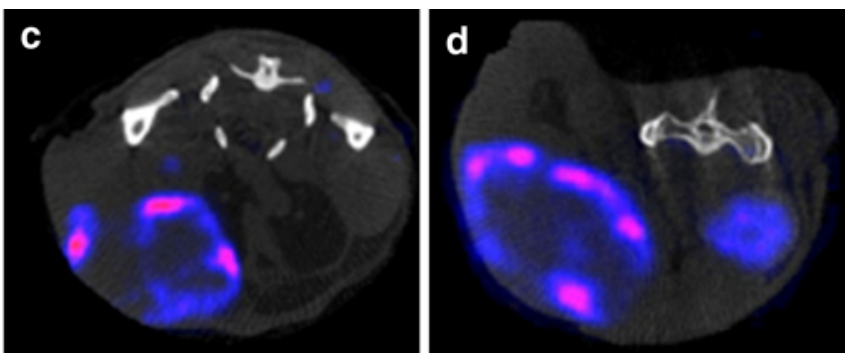

SPECT images for each mouse is shown. After imaging, the tumors were removed, and their weight and uptake of radioactivity were measured (Table 1)

Table 1 Radioactivity accumulation in the tumors that were imaged in vivo with high spatial resolution

\begin{tabular}{|c|c|c|c|c|c|c|}
\hline & \multirow[t]{2}{*}{$\begin{array}{l}\text { Time after } \\
\text { administration }\end{array}$} & \multirow[t]{2}{*}{$\begin{array}{l}\text { Administered } \\
\text { dose (MBq) }\end{array}$} & \multirow[t]{2}{*}{$\begin{array}{l}\text { Tumor } \\
\text { weight }(\mathrm{g})\end{array}$} & \multicolumn{2}{|c|}{$\begin{array}{l}\text { Accumulated radioactivity } \\
\text { in the tumor }\end{array}$} & \multirow{2}{*}{$\begin{array}{l}\text { Tumor uptake } \\
\text { (after decay correction) } \\
\% \text { dose/g }\end{array}$} \\
\hline & & & & $\mathrm{MBq}$ & $\mathrm{MBq} / \mathrm{g}$ & \\
\hline (a) & $48 \mathrm{~h}$ & 8.5 & 0.51 & 0.257 & 0.50 & 9.87 \\
\hline (b) & $48 \mathrm{~h}$ & 8.5 & 0.74 & 0.193 & 0.26 & 5.09 \\
\hline (c) & $48 \mathrm{~h}$ & 15.0 & 1.24 & 0.828 & 0.67 & 9.46 \\
\hline (d) & $24 \mathrm{~h}$ & 12.6 & 1.67 & 2.04 & 1.22 & 9.73 \\
\hline
\end{tabular}

(a)-(d): isolated tumors corresponding to the imaged tumors shown in Fig. 6

$[6,20]$. The best spatial resolution obtained under these conditions was approx. $1.3 \mathrm{~mm}$. It would be acceptable for our purpose, though it did not quite match the best possible performance of the scanner $(1.0 \mathrm{~mm})$. The deterioration in resolution was due to the limited acquisition counts. Therefore, the SPECT requirements for high resolution animal images in vivo are of necessity distinct from those that can provide the best scanner performance.

We also evaluated the lower limit of radioactivity needed for visualization in vivo. We found that radioactivity $<0.1 \mathrm{MBq} / \mathrm{mL}$ could not be visualized on the scanner images under the acquisition conditions described above (Fig. 3). The threshold of radioactivity for visualization was estimated to be $0.2-0.5 \mathrm{MBq} / \mathrm{mL}$ (or g) under the examined conditions. There are few reports on the threshold for visualization with small animal dedicated SPECT scanners. Pissarek et al. [21] reported a threshold level of $0.4-2 \mathrm{MBq} / \mathrm{g}$ of tissue for visualization of mouse brain and heart using $\left[{ }^{99 \mathrm{~m}} \mathrm{Tc}\right]$ hexamethylpropyleneamine oxime, $\left[{ }^{123} \mathrm{I}\right]$ iodophenylpentadecanoic acid, and $\left[{ }^{99 \mathrm{~m}} \mathrm{Tc}\right]$ sestamibi using a Triad 88/Trionix system with three 6-pinhole collimators. Their values were consistent with our results, and the reported image resolution was also similar to ours. Therefore, our threshold values seem to be much to the point. We should note that very good correlation was observed between the SPECT values based on the ROI and the actual radioactivity even for levels $<0.1 \mathrm{MBq} / \mathrm{mL}$, under the visualization threshold.
These results indicate the excellent quantitative performance of the scanner.

It is no easy task to accumulate radioactivity counts in the tumor higher than the threshold level mentioned above. To achieve the necessary level, administration of a substantial amount of radiopharmaceuticals, such as 10-74 MBq/mouse, would be needed. Actually, this and higher amounts of radiopharmaceuticals have been administered in studies on small animal SPECT imaging $[2,11,13,21,22]$. The level is almost the same as the dose per human in conventional clinical SPECT tests. On the other hand, the body weight ratio of a mouse to a human is about 1:2000-3000, and the volume that can be intravenously injected into a mouse is $0.2-0.3 \mathrm{~mL}$ at most. Therefore, radiopharmaceuticals with high specific activity and high concentration are needed [23]. To meet these requirements, we used ${ }^{111}$ In-labeled liposomes. We have previously reported on the preparation of ${ }^{111}$ In-labeled liposomes with high radioactivity and excellent tumor accumulation of the liposomes $[15,16]$. Currently, liposomes are drawing attention as multifunctional nanoparticles for imaging, delivery, and targeting in cancer therapy [12, 24, 25].

The ${ }^{111}$ In-labeled liposomes of $8-15 \mathrm{MBq}$ were administered to the tumor-bearing mice, and their SPECT/ CT images were obtained. As shown in Figs. 4, 5 and 6, the intratumoral distribution of the liposomes was successfully visualized in vivo with good spatial resolution. As shown 
in the serial images of Fig. 5a, the interiors of the tumors were visualized in detail in vivo and were apparently heterogeneous. Comparison of in vivo SPECT images and ARGs (Fig. 5b) confirmed the heterogeneity. The accumulated radioactivity in the tumor that could be imaged was $0.26-1.22 \mathrm{MBq} / \mathrm{mL}$ (Fig. 6), which corresponded well with the threshold for visualization that we established from the phantom experiments (Fig. 3). Previous studies have shown intratumoral heterogeneity by ex vivo ARGs [26-28], but earlier techniques have not enabled in vivo visualization of tumor interiors in detail. The results of the present study are innovative. We have already imaged hypoxia-inducible factor-1-active regions in tumors using our SPECT/CT scanner under the optimal conditions defined here [29].

In conclusion, the optimal conditions required for SPECT imaging with high spatial resolution that enabled visualization of intratumoral heterogeneity in in vivo small animal imaging were investigated, and detailed visualization of tumor interiors using a SPECT scanner was achieved.

Acknowledgments The authors thank Nihon Medi-Physics for their kind donation of ${ }^{111} \mathrm{InCl}_{3}$ solution. This research was partially supported by the grant from Foundation for Promotion Cancer Research, the Health and Labor Sciences Research Grants for Third Term Comprehensive 10-year Strategy for Cancer Control and Grant-in-Aid for Exploratory Research (19659316) from the Ministry of Education, Science, Sports and Culture.

\section{References}

1. Saga T, Koizumi M, Furukawa T, Yoshikawa K, Fujibayashi Y. Molecular imaging of cancer: evaluating characters of individual cancer by PET/SPECT imaging. Cancer Sci. 2009;100(3): 375-81.

2. Beekman FJ, van der Have F, Vastenhouw B, van der Linden AJ, van Rijk PP, Burbach JP, et al. U-SPECT-I: a novel system for submillimeter-resolution tomography with radiolabeled molecules in mice. J Nucl Med. 2005;46(7):1194-200.

3. Rowland DJ, Cherry SR. Small-animal preclinical nuclear medicine instrumentation and methodology. Semin Nucl Med. 2008;38(3):209-22.

4. Franc BL, Acton PD, Mari C, Hasegawa BH. Small-animal SPECT and SPECT/CT: important tools for preclinical investigation. J Nucl Med. 2008;49(10):1651-63.

5. Branderhorst W, Vastenhouw B, van der Have F, Blezer EL, Bleeker WK, Beekman FJ. Targeted multi-pinhole SPECT. Eur J Nucl Med Mol Imaging. 2011;38(3):552-61.

6. Hwang AB, Franc BL, Gullberg GT, Hasegawa BH. Assessment of the sources of error affecting the quantitative accuracy of SPECT imaging in small animals. Phys Med Biol. 2008;53(9): 2233-52.

7. Nuyts J, Vunckx K, Defrise M, Vanhove C. Small animal imaging with multi-pinhole SPECT. Methods. 2009;48(2):83-91.

8. Magota K, Kubo N, Kuge Y, Nishijima K, Zhao S, Tamaki N. Performance characterization of the Inveon preclinical smallanimal PET/SPECT/CT system for multimodality imaging. Eur J Nucl Med Mol Imaging. 2011;38(4):742-52.
9. Acton PD, Kung HF. Small animal imaging with high resolution single photon emission tomography. Nucl Med Biol. 2003;30(8): 889-95.

10. Funk T, Sun M, Hasegawa BH. Radiation dose estimate in small animal SPECT and PET. Med Phys. 2004;31(9):2680-6.

11. Muller C, Forrer F, Schibli R, Krenning EP, de Jong M. SPECT study of folate receptor-positive malignant and normal tissues in mice using a novel ${ }^{99 \mathrm{~m}}$ Tc-radiofolate. J Nucl Med. 2008;49(2): 310-7.

12. Phillips WT, Goins BA, Bao A. Radioactive liposomes. Wiley Interdiscip Rev Nanomed Nanobiotechnol. 2009;1(1):69-83.

13. Chisholm EJ, Vassaux G, Martin-Duque P, Chevre R, Lambert O, Pitard B, et al. Cancer-specific transgene expression mediated by systemic injection of nanoparticles. Cancer Res. 2009;69(6): 2655-62.

14. Cheng D, Wang Y, Liu X, Pretorius PH, Liang M, Rusckowski $\mathrm{M}$, et al. Comparison of ${ }^{18} \mathrm{~F}$ PET and ${ }^{99 \mathrm{~m}} \mathrm{Tc}$ SPECT imaging in phantoms and in tumored mice. Bioconjug Chem. 2010;21(8): $1565-70$.

15. Ogihara-Umeda I, Sasaki T, Kojima S, Nishigori H. Optimal radiolabeled liposomes for tumor imaging. J Nucl Med. 1996; 37(2):326-32.

16. Ogihara-Umeda I, Nishigori H. Radiolabeled liposomes for diagnostic imaging. In: Arshady $\mathrm{R}$, editor. Radiolabeled and magnetic particulates in medicine and biology. London: Citus; 2001. p. $123-48$

17. Ogihara-Umeda I, Kojima S. Increased delivery of gallium-67 to tumors using serum-stable liposomes. J Nucl Med. 1988;29(4): 516-23.

18. Ogihara-Umeda I, Kojima S. Cholesterol enhances the delivery of liposome-encapsulated gallium-67 to tumors. Eur J Nucl Med. 1989;15(9):612-7.

19. Ogihara-Umeda I, Sasaki T, Nishigori H. Development of a liposome-encapsulated radionuclide with preferential tumor accumulation - the choice of radionuclide and chelating ligand. Int J Radiat Appl Instrum B. 1992;19(7):753-7.

20. Mok GS, Tsui BM, Beekman FJ. The effects of object activity distribution on multiplexing multi-pinhole SPECT. Phys Med Biol. 2011;56(8):2635-50.

21. Pissarek M, Meyer-Kirchrath J, Hohlfeld T, Vollmar S, Oros-Peusquens A, Flögel U, et al. Targeting murine heart and brain: visualisation conditions for multi-pinhole SPECT with ${ }^{99 \mathrm{~m}} \mathrm{Tc}$ - and ${ }^{123} \mathrm{I}$-labelled probes. Eur J Nucl Med Mol Imaging. 2009;36(9):1495-509.

22. Forrer F, Valkema R, Bernard B, Schramm NU, Hoppin JW, Rolleman $\mathrm{E}$, et al. In vivo radionuclide uptake quantification using a multi-pinhole SPECT system to predict renal function in small animals. Eur J Nucl Med Mol Imaging. 2006;33(10):1214-7.

23. Kung MP, Kung HF. Mass effect of injected dose in small rodent imaging by SPECT and PET. Nucl Med Biol. 2005;32(7):673-8.

24. Gindy ME, Prud'homme RK. Multifunctional nanoparticles for imaging, delivery and targeting in cancer therapy. Expert Opin Drug Deliv. 2009;6(8):865-78.

25. Sajja HK, East MP, Mao H, Wang YA, Nie S, Yang L. Development of multifunctional nanoparticles for targeted drug delivery and noninvasive imaging of therapeutic effect. Curr Drug Discov Technol. 2009;6(1):43-51.

26. Zhao S, Kuge Y, Mochizuki T, Takahashi T, Nakada K, Sato M, et al. Biologic correlates of intratumoral heterogeneity in ${ }^{18}$ F-FDG distribution with regional expression of glucose transporters and hexokinase-II in experimental tumor. $\mathrm{J}$ Nucl Med. 2005;46(4):675-82.

27. Picchio M, Beck R, Haubner R, Seidl S, Machulla HJ, Johnson $\mathrm{TD}$, et al. Intratumoral spatial distribution of hypoxia and angiogenesis assessed by ${ }^{18} \mathrm{~F}-\mathrm{FAZA}$ and ${ }^{125}$ I-Gluco-RGD autoradiography. J Nucl Med. 2008;49(4):597-605. 
28. Busk M, Horsman MR, Jakobsen S, Keiding S, van der Kogel AJ, Bussink $J$, et al. Imaging hypoxia in xenografted and murine tumors with ${ }^{18} \mathrm{~F}$-fluoroazomycin arabinoside: a comparative study involving microPET, autoradiography, $\mathrm{pO}_{2}$-polarography, and fluorescence microscopy. Int $\mathrm{J}$ Radiat Oncol Biol Phys. 2008;70(4):1202-12.
29. Ueda M, KudoT, Mutou Y, Umeda IO, Miyano A, Ogawa K, et al. Evaluation of $\left[{ }^{125} \mathrm{I}\right] \mathrm{IPOS}$ as a molecular imaging probe for hypoxiainducible factor-1-active regions in a tumor: comparison among SPECT/CT imaging, autoradiography, and immunohistochemistry. Cancer Sci. 2011. doi:10.1111/j.1349-7006.2011.02057.x. 